CDE

March, 2004

\title{
Farsighted Network Formation
}

\author{
Bhaskar Dutta \\ University of Warwick \\ Email:B.Dutta@warwick.ac.uk \\ Sayantan Ghosal \\ University of Warwick \\ Email:S.Ghosal@warwick.ac.uk \\ Debraj Ray \\ University of New York \\ Email: Debraj.Ray@nyu.edu
}

Working Paper No. 122

\section{Centre for Development Economics}

Department of Economics, Delhi School of Economics 
Working Paper No. 122

\title{
Farsighted Network Formation
}

\author{
Bhaskar Dutta \\ Sayantan Ghosal \\ Debraj Ray
}

\begin{abstract}
This paper studies a model of dynamic network formation when individuals are farsighted : players evaluate the desirability of a "current" move in terms of its consequences on the entire discounted stream of payoffs. We define a concept of equilibrium which takes into account far-sighted behavior of agents and allows for limited cooperation amongst agents. We show that an equilibrium process of network formation exists. We also show that there are network structures in which no equilibrium strategy profile can sustain efficient networks. We then provide sufficient conditions under which the equilibrium process will yield efficient outcomes.
\end{abstract}

Keywords: Network formation, farsightedness, equilibrium, efficiency. JEL Classification: C7, D7.

\section{Acknowledgements}

Thanks to Guillanume Haeringer for helpful comments. A version of the paper was written when the first author was visiting the Centre for Development Economics, Delhi School of Economics. 


\section{Introduction}

In several social and economic contexts, the structure of interactions between individuals is best described as a network. The precise structure of interaction across players may be crucial in determining the outcome. Examples include channels of information flow (Bala and Goyal (2000), CalvoArmengol and Jackson (2001), Bramoullé and Kranton (2002) and Kariv (2002)), trading networks (Tesfatsion $(1997,1998)$ and Weisbuch, Kirman and Herreiner(1995)), mutual insurance (Fafchamps and Lund (1997) and Genicot and Ray (2003)), technology adoption (Conley and Udry (2002), Chatterjee and $\mathrm{Xu}$ (2002) and Bandiera and Rasul (2002)) and buyer-seller networks (Kranton and Minehart $(2000,2001)$ and Wang and Watts $(2002)) .{ }^{1}$ Most of these papers explicitly adopt the network formalism, and describe the space of interactions as a graph, where the set of nodes coincides with the set of agents, while an arc between two nodes indicates the existence of bilateral interaction between the corresponding agents.

The theoretical literature on networks, starting from Aumann and Myerson(1988) and Jackson and Wolinsky (1996), emphasizes two related issues. The first issue is the determination of the structure of networks which will be formed if links are established voluntarily by agents so as to maximize individual self-interest, while the second issue is concerned with whether such

\footnotetext{
${ }^{1}$ Dutta and Jackson (2003) contains a collection of papers which examine various issues related to network structures.
} 
endogenous networks are socially efficient.

Following Aumann and Myerson, the typical approach has been to model network formation in a static framework, ${ }^{2}$ though some degree of farsightedness (for instance via offers and counteroffers) can be accommodated even in this context. ${ }^{3}$ A recent departure is Jackson and Watts (2002), which models network formation as an intertemporal process with individuals breaking and forming links as the network evolves dynamically. At the same time, individuals are assumed to act myopically: their decisions are guided completely by current payoffs, although the process of network formation takes place over real time.

Jackson and Watts argue that this form of myopic behaviour makes sense in large networks where players' information may be limited to their immediate "neighborhood", or if players discount the future heavily. To be sure, there would be intrinsic interest in the opposite presumption as well: that agents behave in a farsighted manner and take into account the intertemporal repercussions of their own decisions. A principal aim of this paper is to formalize this idea in the context of network formation.

Our main methodological tool is a variant of the framework introduced by Konishi and Ray (2003) to analyze coalition formation when players are

\footnotetext{
${ }^{2}$ See, for instance, Dutta and Mutuswami (1997), Dutta, van den Nouweland and Tijs (1998), and Slikker and van den Nouweland (2000, 2001).

${ }^{3}$ See, for instance, Currarini and Morelli (2000) and Page, Wooders and Kamat (2001).
} 
farsighted. At first sight, our modification appears to be simply a special case: instead of arbitrary coalitions being active at any date, we only permit a (randomly chosen) pair of agents to be active at the start of a period. However - and here the structure of a network is used to full effect — each agent has natural "unilateral" domains of action. These are the network links that each of the agents has with other agents. An agent can destroy such links unilaterally, and does not require the consent of her partner to do so. At the same time, the pair act together on an equally natural "bilateral" domain. This concerns the formation of a link between the pair. Link formation must be a joint decision. Therefore, while only special "coalitions" can form, they are only enjoined to partly cooperate.

This sort of structure raises interesting conceptual issues, and we cannot pretend to have dealt with them in an entirely satisfactory way. Surely, all actions pertaining to the bilateral link between a pair are commonly observed by the two players, and can therefore serve as correlation devices for their unilateral actions concerning other links. In particular, when a "bilateral deviation" occurs from some ongoing prescribed strategy, both players will be aware of this occurrence. In contrast, we assume that a unilateral deviation by a player that breaks links other than the one with her partner cannot be used as a conditioning device by the partner. We are aware, of course, that this restriction is not entirely satisfactory — we rule out bilateral conditioning on unilateral action - but our own attempts to deal with both types of 
conditioning have led us into difficult terrain (concerning existence, even in mixed strategies) and we have settled for the more modest advance in this paper.

We show in Theorem 1 that a Markovian equilibrium process of network formation exists. An example demonstrates that the equilibrium may necessitate the use of mixed strategies.

We use our solution concept to tackle the question of efficiency in networks. It is well-known that "stable" networks may not be efficient, and the reason for this is simple. When a link is formed, or destroyed, the players involved do so with their own gain in mind. At the same time, these actions also affect the payoff of other players, and so a wedge is driven between stability and efficiency. Theorem 2 restates this in an explicitly dynamic context, using our solution concept: there are network structures where the process will not converge to any efficient network for any equilibrium strategy profile. This is the dynamic counterpart of the conflict between individual incentives and social efficiency demonstrated by Jackson and Wolinsky (1996) for static networks.

A simple way of seeing this conflict (at least for some equilibria) is to study network games where link formation is always profitable in the static sense (to the pair which forms the link). Call this property link monotonicity. Of course, when players $i$ and $j$ form an additional link, some player $k$ may 
suffer a loss in current value. This implies that the complete network is not necessarily socially efficient. Nevertheless, Theorem 3 establishes that there is some equilibrium at which the complete graph is reached in the limit from all initial networks.

Yet other questions remain. For instance, how good is farsighted network formation at resolving "weaker" efficiency issues that stem, for instance, from nonconvexities or increasing returns? In particular, consider situations in which a "small" number of links are costly (to those who form them), while a larger number of links is beneficial to all. Jackson and Watts (2002) observe that myopic agents cannot capture the benefits from such situations: the process may not get off the ground if initial returns are negative. No pair of agents may agree to form the first link if the immediate benefit is smaller than the cost, even if subsequent benefits are exceedingly large.

At first sight, it appears that farsightedness would automatically take care of this problem. A matched pair of agents would surely realize the future gains from linking, even if those benefits are not to be had in the short term. Yet this behavior applied across the board cannot constitute an equilibrium, for then a matched pair would prefer not to form a link until such time as a large number of links have already been built up. This would enable then to save on the transition costs when there are a small number of links. Just because agents are farsighted does not mean that they are impervious to short-term costs. Faced with a less costly transition path they would surely prefer such 
an alternative.

Notice that these efficiency issues are not as weak as coordination failures. There is some element of coordination, in that the efficient outcome is easy enough to sustain as an equilibrium, provided one starts there. But there is also a genuine absence of common interest: starting from the null network, for instance, a player would prefer that other players take the lead in link formation before plunging in herself. These phenomena have been noted in other contexts (see Chamley and Gale (1994) and Adserà and Ray (1998)). Fortunately, we are able to show in Theorem 4 that the complete graph (which must be socially efficient) will be the unique absorbing limit of the network formation process for some equilibrium profile.

Of course, "static" coordination failures can arise even in our dynamic framework. We provide a particularly stark example of this in Example 4, where we show that all matched pairs may break all links at the complete graph even when it is the unique socially efficient network. An implication of such static coordination failures is that typically efficiency cannot be sustained at all equilibria. 


\section{Network Structures}

Let $I$ be a finite index set of $n$ players, and $g$ an undirected graph on $I$. Such a graph, or network, is formally just a collection of $i j$ pairs, the interpretation being that $i$ and $j$ are "linked". ${ }^{4}$ We use the notation $g+i j$ to denote the new graph obtained from $g$ by linking $i$ and $j$.

A component of a network $g$ is a subset $c$ of $g$ such that no $i \in c$ is linked outside $c$ and such that every distinct $i$ and $j$ in $c$ are directly or indirectly linked. ${ }^{5}$ Let $N(c)$ denote the set of individuals who are connected in $c$. Let $C(g)$ denote all the components of $g$.

Let $G$ denote the set of all graphs on all nonempty subsets of $I$. The complete network, denoted $\tilde{g}$, is the graph where all individuals are linked to each other.

Given any graph $g$, and component $c$ in $C(g), w(c, g)$ is the value or total "worth" of players in $c$. The total value of $g$ is

$$
w(g) \equiv \sum_{c \in C(g)} w(c, g)
$$

We will say that $w$ is an additive function if the value of any component $c$ is independent of the structure of links of players not in $c$. In this case, we will

\footnotetext{
${ }^{4}$ Because the graph is undirected, these links are reciprocal. For analyses of network structures which are directed graphs, see Bala and Goyal(2000) and Dutta and Jackson (2000).

${ }^{5}$ Thus isolated singletons are components by definition.
} 
simply write $w(c)$. Notice that an additive function $w$ is a generalization of TU-characteristic functions in cooperative game theory. However, our more general formulation allows for externalities across components of a graph, and so represents a generalization of partition functions since the value of a component depends not only on the coalition structure as in partition functions, but also on how the players in $c$ are linked to each other.

Let $W$ be the set of all worth functions defined on all $(c, g)$ pairs, where $g$ is a network and $c$ a component of $g$.

\subsection{Allocation Rules}

An allocation rule is a mapping $a: G \times W \rightarrow \mathcal{R}^{n}$ such that $\sum_{i \in I} a_{i}(g, w)=$ $w(g)$, for all worth functions $w$ and graphs $g$. The rule specifies the (oneperiod) payoffs to each player $i$ for every conceivable network and worth function. We will refer to the pair $(a, w)$ as a network structure.

An allocation rule satisfies component balance if for all $w \in W$, for all $g \in G$, and for all $c \in C(g), \sum_{i \in I(c)} a_{i}(g, u)=w(c, g)$, where $I(c)$ is the set of agents who appear in $c$. This restriction rules out any cross-subsidization across links.

An allocation rule is anonymous if it distributes payoffs that depend only on player position in the network, and the particular worth function, and not 
player labels. Formally, if $\pi$ is a permutation of $I$, let $g^{\pi}$ be the appropriate transformation of $g$, and for any $w$, define $w^{\pi}$ by $w^{\pi}\left(g^{\pi}\right)=w(g)$. Then $a$ is anonymous relative to $(g, w)$ if for any permutation $\pi, a_{\pi(i)}\left(g^{\pi}, w^{\pi}\right)=a_{i}(g, w)$. Say that the rule is anonymous (without qualification) if it is anonymous relative to every $(g, w)$.

Specifically, the anonymity of $a$ requires that the information used to decide on allocations be obtained only from the function $w$ and the particular network $g$, and not from the label of a player. In particular, observe that the anonymity of an allocation rule implies that individuals who are in symmetric positions in a network are assigned the same allocation, if the underlying $w$ treats such individuals equally, but not necessarily otherwise.

One rule which is both component balanced and anonymous is the componentwise egalitarian allocation rule. This rule distributes worth equally within each component of a graph. That is, letting $a^{e}$ denote the component-wise egalitarian rule, we have

For all $i \in I, a_{i}^{e}(g, w)=\frac{w(c, g)}{|c|}$, where $c \in C(g), i \in I(c)$. 


\subsection{Efficiency}

One can attach different notions of (static) efficiency to network structures. ${ }^{6}$ For instance, efficiency could correspond to maximizing total utility: a graph $g$ is strongly efficient if $w(g) \geq w\left(g^{\prime}\right)$ for all $g^{\prime} \in G$.

A more conservative definition would allow for limited transferability, so that the distribution of payoffs is taken into account. A graph $g$ is (weakly) efficient relative to $(a, w)$ if there is no other $g^{\prime} \in G$ such that $a_{i}\left(g^{\prime}, w\right) \geq$ $a_{i}(g, w)$ for all $i \in I$ with strict inequality for some $j \in I$.

\subsection{Some Restrictions on Network Structures}

Two specific network structures will play a role in what follows. First, a network structure $(a, w)$ exhibits link monotonicity if for all $i, j \in I$, for all $g, a_{i}(g+i j, w)>a_{i}(g, w)$ and $a_{j}(g+i j, w)>a_{j}(g, w)$ whenever $i j \notin g$. Thus, link monotonicity requires that an individual's payoff is increasing in the number of her own links.

To be sure, link monotonicity allows for the possibility that an individual's payoff may go down if other players set up links (not with that player). In particular, the complete network $\tilde{g}$ may not be efficient even when the network structure displays link monotonicity. The example below shows that

\footnotetext{
${ }^{6}$ See Jackson (2001).
} 
when $|N|=3$, the complete network may violate strong efficiency. More complicated examples can be constructed to illustrate the possible violation of (weak) efficiency when $|N| \geq 4$.

ExAmple 1 Let $N=\{1,2,3\}$. The function $w$ is symmetric with $w(\{i j\})=$ $2, w(\{i j, j k\})=7 / 4$, andw $(\tilde{g})=3 / 2$. Moreover, $a_{i}(\{i j\}, w)=a_{j}(\{i j\}, w)=$ $1, a_{i}(\{i j, j k\}, w)=a_{k}(\{i j, j k\}, w)=1 / 4, a_{j}(\{i j, j k\}, w)=5 / 4$, and $a_{l}(\tilde{g}, w)=$ $1 / 2$ for all $l \in N$. Obviously, link monotonicity is satisfied, but the complete network is inefficient.

A network structure $(a, w)$ displays increasing returns to link creation (IRL) if

(i) $w$ is additive and $w(\tilde{g})>0$,

(ii) whenever $c$ is a nonsingleton component of some $g$ with $w(c) \geq 0$, then $w(c)<w\left(c^{\prime}\right)$ for all $c^{\prime} \supset c$,

(iii) if $i \in I(c)$, but $i j \notin g$, then $a_{k}(g+i j, w)>a_{k}(g, w)$ for $k=i, j$.

The definition is complicated to state but the main idea is very simple. A network structure satisfies IRL if along every nested chain of "increasingly connected" networks, there is a threshold (nonempty) network for which the worth turns nonnegative, and both aggregate payoffs as well as payoffs of individuals who form extra links increase as the network becomes larger. 
Of course, link monotonicity and IRL are different conditions. The former applies to all $w$, not just additive ones, while the latter is restricted to the additive case. At the same time, the latter condition only imposes link monotonicity on a subcollection of components, not everywhere, though it also requires that aggregate worth also increase over this subcollection along a nested sequence of components. In particular, IRL guarantees that the complete network is the unique strongly efficient network. In contrast, we have already described an example to show that $\tilde{g}$ may not be strongly efficient when the network structure satisfies link monotonicity.

\section{Some Examples}

In this section, we give some examples to illustrate the framework developed here.

\subsection{The Connections Model.}

This is due to Jackson and Wolinsky (1996). Links represent social relationships. Individuals $i$ and $j$ are "friends" if they are linked together, and friendship is valuable. Individuals also benefit from indirect relationships a "friend of a friend" also brings some benefit. The benefit deteriorates in the 'distance' of the relationship. Let $\pi<1$ be the benefit that $i$ gets from 
a direct link with $j$, and $\pi^{2}$ the benefit that $i$ gets from someone who is at a distance two, and so on. Then,

$$
a_{i}(g, w)=\sum_{i \neq j} \pi^{t(i j)}-\#\{j: i j \in g\} d
$$

where $t(i j)$ is the number of links in the shortest path between $i$ and $j$, and $c$ is the cost per link that $i$ has to pay for each direct link. Here, the total value of a network is simply $w(g)=\sum_{i \in I} a_{i}(g, w)$.

The nature of strongly efficient graphs depend upon the relative values of $\pi$ and $d$. If $d<\pi-\pi^{2}$, then the complete graph $\tilde{g}$ is strongly efficient. In this case, the network structure satisfies both link monotonicity as well as IRL.

A star graph ${ }^{7}$ encompassing all agents is the unique strongly efficient graph for intermediate values of $d$.

If $d>\pi+\left(\frac{N-2}{2}\right) \pi^{2}$, then the empty graph is the unique strongly efficient graph.

\subsection{Group Insurance}

Consider a group of $n$ identical rural communities who are isolated in period zero. In each subsequent period, communities have random endowments. That is, any community $i$ can have a "high" endowment with probability

\footnotetext{
${ }^{7} \mathrm{~A}$ star graph is one where there is a central node to which every other node is connected, and there are no other links.
} 
$p$, and low endowment with the residual probability. These probabilities are independent. Normalize the high endowment to equal one unit and low endowment to equal zero units. Each community is risk-averse, with $v$ being the common increasing, strictly concave utility function.

Any two communities can be connected at a cost of $d$. Here, the connection cost could represent the cost of communication. Any group of connected communities can mutually insure each other. Suppose the insurance contract is that each member of the group will get an equal share of the total realized endowment net of the costs of the links.

Suppose $C(g)=\left\{c_{1}, c_{2}, \ldots, c_{K}\right\}$. With some abuse of notation, let $k$ denote the cardinality of $I\left(c_{k}\right)$. Also, let $d\left(c_{k}\right)$ denote the total cost of network $c_{k}$. Then

$$
w\left(c_{k}\right)=k \sum_{l=0}^{k} p^{l}(1-p)^{k-l}\left(\begin{array}{l}
k \\
l
\end{array}\right) v\left(\frac{l-d\left(c_{k}\right)}{k}\right)
$$

and

$$
a_{i}\left(c_{k}, w\right)=\frac{w\left(c_{k}\right)}{k}
$$

Of course, efficiency requires that each component be minimally connected as long as $d>0$. The properties of the network structure depend on the functional form of the utility function $v$. It is of some interest to identify conditions under which the graph containing all $n$ rural communities is strongly efficient.

Assume that the cost of link formation $d$ is negligibly small, and can be 
ignored. Let $a_{i}(m)$ denote the payoff to $i$ if $i$ is in a component containing $m$ communities. Then

$$
a_{i}(m)=\sum_{k=0}^{m} p^{k}(1-p)^{m-k}\left(\begin{array}{c}
m \\
k
\end{array}\right) v\left(\frac{k}{m}\right) .
$$

We want to identify conditions under which $a_{i}(m)$ is increasing in $m$. Taking a McLaurin series expansion of $v(x)$ around 0 and substituting, we obtain the expression

$$
\begin{aligned}
a_{i}(m)= & \frac{v^{\prime}(0)}{m} \sum_{k=0}^{m} p^{k}(1-p)^{m-k}\left(\begin{array}{c}
m \\
k
\end{array}\right) k+\frac{v^{\prime \prime}(0)}{2 m^{2}} \sum_{k=0}^{m} p^{k}(1-p)^{m-k}\left(\begin{array}{c}
m \\
k
\end{array}\right) k^{2} \\
& +\frac{v^{\prime \prime \prime}\left(\theta \frac{k}{m}\right)}{6 m^{3}} \sum_{k=0}^{m} p^{k}(1-p)^{k}\left(\begin{array}{c}
m \\
k
\end{array}\right) k^{3}
\end{aligned}
$$

If $v^{\prime \prime \prime}(x), 0<x<1$ is small, we can ignore the remainder term to obtain

$$
a_{i}(m)=v^{\prime}(0) p+\frac{v^{\prime \prime}(0)}{2}\left[p^{2}+\frac{p(1-p)}{m}\right]
$$

By assumption, $v^{\prime \prime}(0)<0$. It follows that $a_{i}(m)$ is increasing in $m$. It is easily checked that there are strictly increasing, concave utility functions for which $v^{\prime \prime \prime}(x)$ is small for $0<x<1$. An example is the utility function $v(x)=K x-\frac{x^{2}}{2}, K>1$. Outside $[0,1], v(x)$ can always be extended so that it continues to be strictly increasing and concave. However, in general, $a_{i}(m)$ is not necessarily increasing in $m$. 


\subsection{Networks of Collaboration in Oligopoly.}

This is due to Goyal and Joshi (2003). ${ }^{8}$ Consider an oligopoly setting where firms form pairwise collaborative links with other firms. The collaboration could involve joint research activities, sharing knowledge about markets, sharing facilities such as distribution channels. A collaboration link between firms $i$ and $j$ yields lower costs of production for the two firms. Any collaboration network thus induces a distribution of costs across firms. Given these costs, firms subsequently compete on the product market as Cournot oligopilists.

Assume that initially all firms have zero fixed costs, and constant returnsto-scale cost functions. The initial marginal cost of firm $i$ is $c_{i 0}$. Given a graph $g$, let $\mu_{i}(g)$ denote the number of firms with which $i$ has collaboration links in $g$. Then, the resulting marginal cost of firm $i$ is:

$$
c_{i}(g)=c_{i 0}-\gamma \mu_{i}(g) .
$$

where $\gamma>0$ is the cost reduction induced by each link formed by a firm. ${ }^{9}$

Suppose the inverse market demand curve is linear :

$$
p=a-q
$$

The output produced by firm $i$ in the Cournot game will be:

$$
q_{i}(g)=\frac{\left(a-n c_{i 0}+\sum_{j \neq i} c_{j 0}\right)+n \gamma \mu_{i}(g)-\gamma \sum_{j \neq i} \mu_{j}(g)}{n+1}
$$

\footnotetext{
${ }^{8}$ See also Goyal and Moraga(2001).

${ }^{9}$ Assume that $\gamma$ is small, so that net marginal cost is always positive for each firm.
} 
The profit will be $\left(q_{i}(g)\right)^{2}$. Notice that the profit of firm $i$ increases if $i$ sets up an additional link with say firm $j$. While this additional link also reduces firm $j$ 's cost, the overall effect on firm $i$ 's profit is positive. Hence, the network structure satisfies link monotonicity. On the other hand, an additional link by two rival firms $k$ and $l$ reduces firm $i$ 's profit. So, the network structure does not satisfy IRL.

\section{Process of Network Formation}

Suppose that at any date, a pair of players $i$ and $j$ is randomly chosen (with uniform probability) and endowed with the capacity to take actions at that date. Each of these players can unilaterally sever any existing link with any other player, and they can bilaterally form a link between the two of them if one doesn't exist to begin with. These actions create a (possibly) new graph, and then one-period payoffs are received according to the given allocation rule. The current period then ends, and the whole process begins again ad infinitum.

Thus there are two components of a strategy in force: unilateral, which involves link severance, and bilateral, which involves link creation (and will generally be correlated across the relevant actors). Throughout, we will assume that players follow Markov strategies; i.e., their actions will be presumed to depend only on the existing payoff-relevant state. 
Because strategies involve some elements of correlation and independence, we will need to be more specific and careful in describing them. Suppose that two individuals "partially cooperate", as they do here in setting up a bilateral link, but also take independent actions, as they do here with link destruction. Then all matters related to the bilateral link between $i$ and $j$ are commonly observed by the two players, and can therefore serve as correlation devices: either player can condition his other unilateral actions on the fate of this link. For instance, when a "bilateral deviation" occurs from some ongoing prescribed strategy, both players will be aware of this and can condition their independent actions on such deviation. In contrast, a unilateral deviation by $i$ that breaks links other than the one with $j$ cannot be used as a conditioning device by $j$. This suggests that the situation is formally equivalent to one in which (at any date) actions pertaining to the $i j$ link are taken "first" and these are "followed" by the unilateral actions pertaining to all other existing links. ${ }^{10}$ Let us make this approach more formal.

It will be useful to define a principal state as a collection $s=(g, i j)$, where $g$ is the historically given graph and $i j$ is the chosen active pair. Define an intermediate state as a collection $s=(g, i j, \zeta)$, where $g$ and $i j$ are as before, and $\zeta$ is a variable which takes the value 0 if the pair $i j$ is not linked, and the value 1 if $i j$ is linked. An intermediate state doesn't physically exist; it is a conceptual halfway point for defining unilateral actions; hence the choice

\footnotetext{
${ }^{10}$ The phrases that suggest chronology are deliberately in quotes because no real chronology is implied.
} 
of terminology. In contrast, a principal state physically exists at the start of a period. When there is no need for a distinction, we shall simply use "state" to denote either of the two varieties. Notice, too, that we use the same notation $s$ which will also ease the writing.

For any intermediate state $s=(g, i j, \zeta)$, define $D_{i}(s) \equiv\{k \neq j \mid i k \in g\}$, and likewise define $D_{j}(s)$. These are the sets of existing linkages to $i$, but never counting $j$. As already discussed, a bilateral action to create (or maintain) the link between an active pair is not undone at the intermediate state. This does not mean that unilateral breaking of an $i j$-link is not permitted; it certainly is but only at the principal state. Put another way, such actions are commonly experienced and can serve as correlating devices for actions on the "unilateral domains" $D_{i}(s)$ and $D_{j}(s)$.

Moreover, note that by assumption, $i$ and $j$ can break links on their unilateral domains; no links other than those pertaining to the active pair can be created during the period.

Formally, then, (mixed) actions may be described as follows. At any principal state $s$ with active pair $i j$ it is simply a probability $\mu(s)=q$ of bilateral linkage between $i$ and $j$. At any intermediate stage $s$ with active pair $i j$ it is a collection $\mu(s) \equiv\left\{\nu_{i}, \nu_{j}\right\}$, where for each $k=i, j, \nu_{k}$ is a probability measure defined over all subsets (including the empty subsets) of $D_{k}(s) .{ }^{11}$ We will let

\footnotetext{
${ }^{11}$ As a matter of notation, we should also index the individual $\nu$-components by $s$, but this is notationally cumbersome and hopefully the context will prevent any confusion.
} 
$\boldsymbol{\mu}$ stand for the entire profile of $\mu(s)$ 's over all states (notice that $\mu(s)$ has a different interpretation depending on what sort of state we are looking at), and refer to $\boldsymbol{\mu}$ as a strategy profile.

A strategy profile precipitates - for each state $s$, principal or intermediate — some probability measure $\lambda_{s}$ over the feasible set $F(s)$ of future networks starting from $s$. In particular, a Markov process is induced on the set $S$ of principal states: at any principal state $s, \lambda_{s}$ describes the movement to a new network, and the given random choice of active players moves the system to a new active pair.

The process creates values for each player. Assuming that the $a_{k}$ 's are vN-M payoffs, we can write - for every state $s$ with active pair $i j$ - the overall payoff to any person $k$ (under the strategy profile $\boldsymbol{\mu}$ ) as the unique solution to the functional equation

$$
V_{k}(s, \boldsymbol{\mu})=\sum_{g^{\prime} \in F(s)} \lambda_{s}\left(g^{\prime}\right)\left[a_{i}\left(g^{\prime}\right)+\delta_{i} \sum_{i^{\prime} j^{\prime}} \pi\left(i^{\prime} j^{\prime}\right) V_{k}\left(s^{\prime}, \boldsymbol{\mu}\right)\right]
$$

where $\delta_{i} \in(0,1)$ is the discount factor of agent $i, \lambda_{s}$ is the probability over $F(s)$ associated with $\boldsymbol{\mu}, \pi\left(i^{\prime} j^{\prime}\right)$ is the probability that a pair $i^{\prime} j^{\prime}$ will be active "tomorrow", and $s^{\prime}$ stands for the principal state $\left(g^{\prime}, k^{\prime} \ell^{\prime}\right)$. [Note that $V_{k}$ is well-defined on both principal and intermediate states.]

We will also find it convenient to denote $V_{k}(g, \boldsymbol{\mu})$ as the (expected) payoff 
to $k$ at a given network $g$. This is given by

$$
V_{k}(g, \boldsymbol{\mu})=\frac{2}{n(n-1)} \sum_{i j \in I \times I} V_{k}((g, i j), \boldsymbol{\mu})
$$

\subsection{Equilibrium}

Loosely speaking, an equilibrium process of network formation is a strategy profile $\boldsymbol{\mu}$ with the property that there is no active pair at any state $s$ which can benefit - either unilaterally or bilaterally - by departing from $\mu(s)$. The benefit is evaluated according to the value function introduced above. The remainder of this section contains a precise formulation of this idea. Before the formalities are introduced, however, note the following points:

[1] Profitable deviations are not necessarily myopic: individuals take the ongoing process as given and evaluate the entire stream of consequences arising from a single action. One can imitate perfectly myopic behavior by taking the discount factor to zero, and perfect farsightedness by taking the opposite limit.

[2] Network formation and payoffs occur together. There is no "waiting" in the model until some "stable" network is formed, following which payoffs are assigned. Indeed, our definition permits cycles and continued flux in the network, and there is no difficulty at all in evaluating overall payoffs. 
Now for a precise account. Fix some ongoing strategy profile $\boldsymbol{\mu}$ and an intermediate state $s$ with active pair $i j$. A unilateral move for $i$ at $s$ (to be sometimes referred to as an $i$-unilateral move at $s$ when it's necessary to keep track of the relevant agent) is simply a collection $\mu^{\prime}(s)=\left\{\nu_{i}^{\prime}, \nu_{j}\right\}$, where the $j$ th component cannot be altered from that of $\mu(s)$. (Likewise for $j$.) Given a principal state $s$, a bilateral move for the active pair $i j$ is a new probability $\mu^{\prime}(s)$ of $i j$-linkage. A unilateral move for $i$ at the principal state $s$ is also a new probability $\mu^{\prime}(s)$ of $i j$-linkage, but it "takes effect" only if the $i j$-linkage is present in the historically given graph. That is, $i$ can unilaterally decide to alter the probability of the $i j$-linkage provided $i$ and $j$ are already linked together.

In words, unilateral moves for an agent can either destroy $i j$-linkage and/or other existing linkages on that agent's unilateral domain. Of course, an $i$ unilateral move cannot prescribe changes on $j$ 's unilateral domain. A bilateral move can only create $i j$-linkage (everything else belongs to the unilateral domains). Now, in part this compartmentalization is a matter of semantics. For instance, suppose that at some principal state $s=(g, i j), i j$ is unlinked for sure; that is, $q(s)=0$. So the intermediate state $s^{\prime}=(g, i j, 1)$ will never happen. Nevertheless, the actions $\mu\left(s^{\prime}\right)=\left(\nu_{i}, \nu_{j}\right)$ are still specified, even though they don't kick in under $\boldsymbol{\mu}$. Now if a bilateral move or "deviation" links $i j$, the play will generally change on other fronts as well, as the unilateral action pairs "switch" from $\mu(g, i j, 0)$ to $\mu(g, i j, 1)$. More on these matters 
below.

Now, fix some profile $\boldsymbol{\mu}$ and a state $s$ (principal or intermediate). For any $\mu^{\prime}(s)$ and for each player $k$, define

$$
V_{k}\left(s, \boldsymbol{\mu}, \mu^{\prime}(s)\right) \equiv \sum_{g^{\prime} \in F(s)} \lambda_{s}^{\prime}\left(g^{\prime}\right)\left[a_{k}\left(g^{\prime}\right)+\delta_{i} \sum_{i^{\prime} j^{\prime}} \pi\left(i^{\prime} j^{\prime}\right) V_{k}\left(s^{\prime}, \boldsymbol{\mu}, \mu^{\prime}(s)\right)\right]
$$

where $s^{\prime}$ is the principal state $\left(g^{\prime}, i^{\prime} j^{\prime}\right)$. For an intermediate state $s$ with active pair $i j$, and for some $k=i, j$, say that a $k$-unilateral move $\mu^{\prime}(s)$ is profitable if

$$
V_{k}\left(s, \boldsymbol{\mu}, \mu^{\prime}(s)\right)>V_{k}(s, \boldsymbol{\mu}, \mu(s)),
$$

Likewise, for a principal state $s$ with active pair $i j$, say that a bilateral move $\mu^{\prime}(s)$ is profitable if

$$
V_{i}\left(s, \boldsymbol{\mu}, \mu^{\prime}(s)\right)>V_{i}(s, \boldsymbol{\mu}) \text { and } V_{j}\left(s, \boldsymbol{\mu}, \mu^{\prime}(s)\right)>V_{j}(s, \boldsymbol{\mu}) .
$$

A strategy profile $\boldsymbol{\mu}$ is an equilibrium if at no $s$ is a unilateral or bilateral move profitable.

Notice how our description of equilibrium subsumes a rationality requirement akin to perfection. ${ }^{12}$ An equilibrium must be immune to all profitable moves, including those starting from intermediate states that may never be reached.

\footnotetext{
${ }^{12}$ It is not the same as perfection because — properly speaking — an intermediate stage may not be viewable as a subgame. It is a device designed to capture partial cooperation.
} 


\subsection{Existence}

One can establish the following

THEOREM 1 An equilibrium in mixed bilateral and unilateral strategies always exists.

Proof. For every state $s$ look at the space $U(s)$ of all possible $\mu(s)$. Let $\mathbf{U} \equiv \prod_{s \in S} U(s)$. [Note: with the obvious product topology, $\mathbf{U}$ is viewable as a compact, convex subset of some finite-dimensional Euclidean space.] For each $s$, we construct a nonempty-valued, convex-valued uhc correspondence $\Psi_{s}$ from $\mathbf{U}$ to $U(s)$ in the following way.

Fix some $\boldsymbol{\mu} \in \mathbf{U}$, and consider any state $s$. If $s$ is an intermediate state with active pair $i j$, maximize - for each $k \in\{i j\}$ - the value of $V_{k}\left(s, \boldsymbol{\mu}, \mu^{\prime}(s)\right)$ over all $k$-unilateral deviations from $s$. Gather all the mixed actions $\nu_{k}^{\prime}$ that achieve this maximum. Because $V_{k}$ is linear in $\nu_{k}^{\prime}$, this collection is nonempty and convex: call it $A_{k}(s, \mu)$. Define $\Psi_{s}(\boldsymbol{\mu}) \equiv A_{i}(s, \mu) \times A_{j}(s, \mu)$.

We've already seen that $\Psi_{s}$ is a nonempty- and convex-valued correspondence. The fact that it is uhc follows from the continuity of $V_{k}\left(s, \boldsymbol{\mu}, \mu^{\prime}(s)\right)$ in $\boldsymbol{\mu}$ and the maximum theorem. 
If $s$ is a principal state with active pair $i j$, then denote by $s^{0}$ and $s^{1}$ the two intermediate states that emerge from it. If the expression

$$
\min \left\{V_{i}\left(s^{1}, \boldsymbol{\mu}\right)-V_{i}\left(s^{0}, \boldsymbol{\mu}\right), V_{j}\left(s^{1}, \boldsymbol{\mu}\right)-V_{j}\left(s^{0}, \boldsymbol{\mu}\right)\right\}
$$

is strictly positive, define $\Psi_{s}(\boldsymbol{\mu})=\{1\}$. If the expression is strictly negative, set $\Psi_{s}(\boldsymbol{\mu})=\{0\}$. If it is zero, let $\Psi_{s}(\boldsymbol{\mu})=[0,1]$.

It is obvious that for each $\boldsymbol{\mu}, \Psi_{s}(\boldsymbol{\mu})$ is nonempty and convex. Using the continuity of $V_{k}\left(s^{\prime}, \boldsymbol{\mu}\right)$ in $\boldsymbol{\mu}$, it is straightforward to check that $\Psi_{s}(\boldsymbol{\mu})$ is uhc.

It follows from the two previous paragraphs and Kakutani's theorem that the product $\Psi \equiv \prod_{s \in S} \Psi_{s}$ (which is a correspondence from $\mathbf{U}$ to $\mathbf{U}$ ) must have a fixed point $\boldsymbol{\mu}^{*}$ in $\mathbf{U}$. By construction, no profitable deviation - unilateral or bilateral - is possible from $\boldsymbol{\mu}^{*}$, and therefore it is an equilibrium.

Of course, the theorem establishes the existence of equilibrium in mixed strategies. This qualification is important because the next example shows that an equilibrium in pure strategies may not always exist.

Example 2: Let $I=\{1,2,3\}$. Consider the allocation rule defined below.

(i) $a_{1}\left(g^{1}\right)=a, a_{2}\left(g^{1}\right)=b$, where $g^{1}=\{12\}$, and $a=b+\epsilon$.

(ii) $a_{2}\left(g^{2}\right)=a, a_{3}\left(g^{2}\right)=b$ where $g^{2}=\{23\}$.

(iii) $a_{3}\left(g^{3}\right)=a, a_{1}\left(g^{3}\right)=b$ where $g^{3}=\{13\}$. 
(iv) On all other graphs $g$ with at least one arc, $w(g)$ is a large negative number, and a distributes the (negative) value equally.

Suppose $\boldsymbol{\mu}$ is a pure strategy equilibrium. We first show that there cannot be any absorbing graph. ${ }^{13}$ Wlog, suppose $g^{1}$ is an absorbing graph. Consider the principal state $s=\left(g^{3}, 12\right)$. Then, since $a>b$, we must have $\lambda_{s}\left(g^{1}\right)=1$. To check this assertion, note that if the assertion is not true, then either $V_{1}\left(g^{3}, \boldsymbol{\mu}\right) \geq \frac{a}{1-\delta}$ or $V_{2}\left(g^{3}, \boldsymbol{\mu}\right) \geq \frac{b}{1-\delta}$. Since $V_{1}\left(g^{3}, \boldsymbol{\mu}\right)$ must be strictly less than $\frac{a}{1-\delta}$ if $\lambda_{s}\left(g^{1}\right)=0$, the only possibility is that $V_{2}\left(g^{3}, \boldsymbol{\mu}\right) \geq \frac{b}{1-\delta}$. This possibility too can be ruled out.

This in turn implies that $g^{2}$ is also an absorbing graph since neither 2 nor 3 want to move from $g^{2}$. But, if $g^{2}$ is an absorbing graph, then we must have $\lambda_{s}\left(g^{2}\right)=1$ where $s=\left(g^{1}, 23\right)$ since $a>b$. This contradicts the initial supposition that $g^{1}$ is an absorbing graph.

The only other possibility is that $\left\{g^{1}, g^{2}, g^{3}\right\}$ form a closed set. In this case, we have $\lambda_{s}\left(g^{2}\right)=1$ where $s=\left(g^{1}, 23\right), \lambda_{s^{\prime}}\left(g^{3}\right)=1$ where $s^{\prime}=\left(g^{2}, 13\right)$, and $\lambda_{\bar{s}}\left(g^{1}\right)=1$ where $\bar{s}=\left(g^{3}, 12\right)$. Then, the following are true:

$$
\begin{aligned}
V_{2}(s, \boldsymbol{\mu}) & =a+\delta V_{2}\left(g^{2}, \boldsymbol{\mu}\right) \\
V_{2}\left(g^{2}, \boldsymbol{\mu}\right) & =\frac{1}{3}\left[V_{2}\left(\left(g^{2}, 12\right), \boldsymbol{\mu}\right)+V_{2}\left(\left(g^{2}, 13\right), \boldsymbol{\mu}\right)+V_{2}\left(\left(g^{2}, 23\right), \boldsymbol{\mu}\right)\right] \\
& =\frac{2 a}{3-2 \delta}+\frac{\delta V_{2}\left(g^{3}, \boldsymbol{\mu}\right)}{3-2 \delta}
\end{aligned}
$$

\footnotetext{
${ }^{13}$ A graph $g$ is absorbing if $\lambda_{s}(g)=1$ for all states $s=(g, i j)$.
} 


$$
\begin{aligned}
V_{2}\left(g^{3}, \boldsymbol{\mu}\right) & =\frac{1}{3}\left[V_{2}\left(\left(g^{3}, 12\right), \boldsymbol{\mu}\right)+V_{2}\left(\left(g^{3}, 13\right), \boldsymbol{\mu}\right)+V_{2}\left(\left(g^{3}, 23\right), \boldsymbol{\mu}\right)\right] \\
& =\frac{b}{3-2 \delta}+\frac{\delta V_{2}\left(g^{1}, \boldsymbol{\mu}\right)}{3-2 \delta} \\
V_{2}\left(g^{1}, \boldsymbol{\mu}\right) & =\frac{1}{3}\left[V_{2}\left(\left(g^{1}, 12\right), \boldsymbol{\mu}\right)+V_{2}\left(\left(g^{1}, 13\right), \boldsymbol{\mu}\right)+V_{2}(s, \boldsymbol{\mu})\right] \\
& =\frac{2 b}{3-2 \delta}+\frac{V_{2}(s, \boldsymbol{\mu})}{3-2 \delta}
\end{aligned}
$$

Making appropriate substitutions and simplifying,

$$
V_{2}(s, \boldsymbol{\mu})=\frac{3 a}{(3-2 \delta)\left(1-\frac{\delta^{3}}{(3-2 \delta)^{3}}\right)}+\frac{\delta^{2} b}{(1-\delta)\left((3-2 \delta)^{2}+\delta^{2}+(3-2 \delta) \delta\right)}
$$

On the other hand, if 2 deviates at the principal state $s$ and refuses to form the link with 3 , then $g^{1}$ would be an absorbing graph, and then $V_{2}\left(s,\left(\boldsymbol{\mu}_{2}, \mu_{2}^{\prime}\right)\right)=$ $\frac{b}{1-\delta}$. We show that for $\delta$ large enough, $\frac{b}{1-\delta}>V_{2}(s, \boldsymbol{\mu})$, thereby contradicting the supposition that $\boldsymbol{\mu}$ is an equilibrium. To this end, note that

$$
\frac{V_{2}(s, \boldsymbol{\mu})}{\frac{b}{1-\delta}}=\frac{3 a(1-\delta)}{b(3-2 \delta)\left(1-\left(\frac{\delta}{3-2 \delta}\right)^{3}\right)}+\frac{\delta^{2}}{\left.(3-2 \delta)^{2}+\delta^{2}+(3-2 \delta) \delta\right)}
$$

Using L'Hospital's rule, $\lim _{\delta \rightarrow 1} \frac{1-\delta}{\left(1-\frac{2}{3} \delta\right)\left(1-\left(\frac{\delta}{3-2 \delta}\right)^{3}\right)}=\frac{1}{3}$. Also, $\lim _{\delta \rightarrow 1} \frac{\delta^{2}}{\left.(3-2 \delta)^{2}+\delta^{2}+(3-2 \delta) \delta\right)}=$ $\frac{1}{3}$. Hence,

$$
\lim _{\delta \rightarrow 1} \frac{V_{2}(s, \boldsymbol{\mu})}{\frac{b}{1-\delta}}<1 \text { when } \frac{b}{a}<2
$$

This establishes that $\boldsymbol{\mu}$ is not an equilibrium since $\epsilon$ can be chosen smaller than $b$. 


\section{Sustaining Efficiency}

An issue in this general setting that merits special attention is the question of network efficiency. It hardly needs mentioning that the efficiency question is fundamental: the "Coase theorem" tells us that under free and unrestrained negotiation, with the ability to write unlimited binding agreements, the equilibrium outcome must be efficient. Whether or not the "Coase Theorem" is true is a complex question: it depends on how one models "free and unrestrained negotiation": there are conceptual difficulties in a precise description of what exactly this phrase means. A more modest goal is to describe small departures from the unrestrained ideal, and see how much of an efficiency failure (if at all) takes place.

The great merit of the current setup is that it captures one central aspect of free negotiation, which has to do with continued renegotiation. In this framework, links can be altered continually, and if there is little discounting of the future, this means that there is essentially vast scope for continued movement with very little cost. Does this give rise to efficient outcomes? Our results suggest that the answer to this question is far from easy.

We have already provided alternative definitions of efficient networks. There is then the question of what it means for network formation processes to yield these efficient outcomes. These have to do with concepts of absorption: an efficient state (however defined) may be strongly absorbing (in the 
sense that it attracts the process from all initial conditions), or it may be just a stationary state (other weak definitions, such as recurrence, are also possible).

The most satisfactory situation is one in which all equilibrium strategy profiles result in transition probability matrices which have the property that all closed sets are contained in the set of efficient graph(s). This would ensure that even if mistakes are made along the way ${ }^{14}$ the process of network formation converges to some efficient graph(s) with probability one. Of course, this is an extremely strong requirement, and one that we should not normally expect to be satisfied. For instance, even if players are farsighted, there is no reason why they should be able to ensure that "static" coordination failures are avoided.

In order to demonstrate that the issue of sustaining efficiency in this framework is not a trivial proposition, we also show that there are network structures in which no equilibrium strategy profile can sustain any efficient network as a strongly absorbing state. This can be viewed as the dynamic counterpart of the conflict between (static) stability and efficiency demonstrated by Jackson and Wolinsky (1996). To show this, say that an allocation rule allows limited transfers if $a_{i}(g, w) \leq w(g)$ for all $i$ whenever $w(g) \geq 0$.

An allocation rule which allows limited transfers does not permit other

\footnotetext{
${ }^{14}$ By "mistakes" we mean non-equilibrium behavior. If mistakes are not made, then it is sufficient to consider whether all paths starting from the empty graph converge to some efficient graph.
} 
individuals to "overcompensate" any individual.

THEOREM 2 Let the allocation rule a be anonymous and allow limited transfers. Then there is $w$ and $\bar{\delta}<1$ such that for all $\delta \in(\bar{\delta}, 1)$ no efficient graph (relative to $(a, w))$ is strongly absorbing at any pure strategy equilibrium profile.

Proof. Let $I=\{1,2,3\}$. Choose $w$ such that $w\{i j\}=2 \alpha, w\{i j, i k, j k\}=$ $3 \alpha, w\{i j, j k\}=0$, where $\alpha>0$. Since $a$ is anonymous, $a_{k}(\{i j\})=\alpha$ for $k=i, j$. Similarly, each agent gets $\alpha$ at the complete graph. Finally, the limited transfers property ensures that each agent gets 0 at $\{i j, j k\}$.

The unique efficient graph here is the complete graph. We want to show that there is no pure strategy equilibrium at which $\tilde{g}$ is strongly absorbing. ${ }^{15}$

Suppose instead that $\mu^{*}$ is a pure strategy equilibrium for which $\tilde{g}$ is strongly absorbing. Since all subsequent statements are with reference to this strategy profile, we will simply write $V_{i}(g)$, etc., instead of $V_{i}\left(g, \mu^{*}\right)$.

We also use the notation $\{i j\} \rightarrow_{i k} g$ to denote that the network $\{i j\}$ is changed to $g$ at the principal state $(\{i j\}, i k)$ according to $\mu^{*}$.

Then, the following are true:

\footnotetext{
${ }^{15}$ However, there are pure strategy equilibria in which $\tilde{g}$ and each of the one-link graphs are absorbing.
} 
(F1) $\{i j\} \rightarrow_{i j}\{i j\}$.

(F2) $\{i j\} \rightarrow_{i k} g \neq\{i j\}$ and $\{i j\} \rightarrow_{j k} g^{\prime} \neq\{i j\}$ for $k \notin\{i, j\}$.

Note that if (F2) does not hold at some $\{i j\}$, then $\{i j\}$ must be an absorbing graph at any equilibrium, which contradicts the supposition that $\tilde{g}$ is strongly absorbing.

If $\tilde{g}$ is to be strongly absorbing, then there must exist some principal state $s=(\{i j\}, i k)$ such that $\{i j\} \rightarrow_{i k}\{i j, i k\}$. Wlog, let $s=(\{12\}, 13)$, and denote $g=\{12,13\}$. Then,

$$
V_{1}(s)=\delta V_{1}(g)=\frac{\delta \alpha}{(3-2 \delta)(1-\delta)}
$$

Case 1: Suppose $\{12\} \rightarrow_{23}\{12,23\}=g^{\prime}$.

Suppose 1 deviates from $\mu^{*}$ at $s$ by refusing the link with 3 , but retains the link with 2. Denoting the resulting discounted payoffs by $V_{1}^{\prime}$,

$$
\begin{aligned}
V_{1}^{\prime}(s) & =\alpha+\delta V_{1}^{\prime}(\{12\}) \\
& =\alpha+\frac{\delta}{3}\left[2 \alpha+2 \delta V_{1}^{\prime}(\{12\})+\delta V_{1}^{\prime}\left(g^{\prime}\right)\right] \\
& =\frac{3 \alpha+\delta^{2} V_{1}^{\prime}\left(g^{\prime}\right)}{3-2 \delta}
\end{aligned}
$$

Using $V_{1}\left(g^{\prime}\right)=V_{1}(g)$,

$$
V_{1}^{\prime}(s)-V_{1}(s)=\frac{3 \alpha}{3-2 \delta}+\frac{\delta \alpha}{(3-2 \delta)(1-\delta)}\left(\frac{\delta}{3-2 \delta}-1\right)
$$




$$
\begin{aligned}
& =\frac{3 \alpha}{3-2 \delta}-\frac{3 \alpha \delta}{(3-2 \delta)^{2}} \\
& >0
\end{aligned}
$$

But, then $\mu^{*}$ cannot be an equilibrium.

Case 2: Suppose $\{12\} \rightarrow_{23}\{23\} .{ }^{16}$

We let the reader check that if $\{23\} \rightarrow_{13}\{13\}$, then 2 is better off by retaining the link with 1 and refusing to form the link with 3 at $s^{\prime}=(\{12\}, 23)$.

So, we only need to check for the case $\{23\} \rightarrow_{13}\{13,23\}=g^{\prime \prime}$ and $\{23\} \rightarrow_{12}\{12\} \cdot{ }^{17}$

In this case, we have to consider specifications of $\mu^{*}$ at principal states involving the network $\{13\}$.

Case 2(a): Suppose $\{13\} \rightarrow_{12}\{12\}$ and $\{13\} \rightarrow_{23}\{13,23\}$.

Then,

$$
V_{1}(\{13\}, 12)=\alpha+\delta V_{1}(\{12\})
$$

where

$$
V_{1}(\{12\})=\frac{\alpha}{3-2 \delta}+\frac{\delta V_{1}(g)}{3-2 \delta}
$$

\footnotetext{
${ }^{16}$ In view of (F2), this is the only remaining possibility.

${ }^{17}$ If $\{23\} \rightarrow_{13}\{13,23\}$, then it cannot be the case that $\{23\} \rightarrow_{12}\{12,23\}$. Simply apply the proof of Case 1 to establish that 2 will then have a profitable deviation at the principal state $(\{23\}, 12)$.
} 
Suppose 1 deviates from $\mu^{*}$ at the principal state $(\{13\}, 12)$, by retaining the link with 3 and refusing to form the link with 2. Denoting the resulting discounted payoffs by $V^{\prime}$,

$$
V_{1}^{\prime}\left((\{13\}, 12)=\alpha+\delta V_{1}^{\prime}(\{13\}) .\right.
$$

But

$V_{1}^{\prime}(\{13\})=\frac{2 \alpha}{3-2 \delta}+\frac{\delta}{3-2 \delta} V_{1}(\{13,23\})=\frac{2 \alpha}{3-2 \delta}+\frac{\delta \alpha}{(3-2 \delta)^{2}(1-\delta)}>V_{1}(\{12\})$.

Hence, $\mu^{*}$ cannot be an equilibrium in this case.

Case 2(b): Suppose $\{13\} \rightarrow_{12}\{12,13\}$ and $\{13\} \rightarrow_{23}\{23\}$

In this case, 3 has a profitable unilateral deviation at $(\{13\}, 23)-3$ can retain link with 1 and refuse to form link with 2 .

Case 2(c): Suppose $\{13\} \rightarrow_{12}\{12\}$ and $\{13\} \rightarrow_{23}\{23\}$.

Then,

$$
V_{3}(\{13\}, 23)=\alpha+\delta V_{3}(\{23\}) .
$$

Also,

$$
\begin{aligned}
& V_{3}(\{23\})=\frac{\alpha}{3-\delta}+\frac{\delta}{3-\delta} V_{3}(\{13,23\})+\frac{\delta}{3-\delta} V_{3}(\{12\}) . \\
& V_{3}(\{12\})=\frac{\alpha}{3-\delta}+\frac{\delta}{3-\delta} V_{3}(\{23\})+\frac{\delta}{3-\delta} V_{3}(\{12,13\}) .
\end{aligned}
$$


Using the fact that $V_{3}(\{12,13\})=V_{3}(\{13,23\})=\frac{\delta \alpha}{(3-2 \delta)(1-\delta)}$, and simplifying,

$$
V_{3}(\{23\})=\frac{\alpha}{3-2 \delta}+\frac{\delta}{3-2 \delta} V_{3}(\{13,23\})
$$

Hence,

$$
V_{3}(\{13\}, 23)=\frac{3 \alpha-\alpha \delta}{3-2 \delta}+\frac{\delta^{2}}{3-2 \delta} V_{3}(\{13,23\}) .
$$

Now, suppose 3 deviates at the intermediate state so that after forming a link with 2,3 retains the link with 1 . Then,

$$
V_{3}^{\prime}(\{13\}, 23)=\delta V_{3}(\{13,23\})
$$

Hence,

$$
V_{3}^{\prime}(\{13\}, 23)-V_{3}(\{13\}, 23)=\frac{3 \alpha \delta}{(3-2 \delta)^{2}}-\frac{3 \alpha-\alpha \delta}{3-2 \delta} .
$$

This is positive for $\delta$ large enough.

Also, note that 2 is better off forming the link with 3 rather than remaining at $\{13\}$, even if 3 refuses to cut the link with 1 . Hence, $\mu^{*}$ cannot be an equilibrium in this case either.

Using (F2), this exhausts all possible cases, and so establishes the theorem.

Notice that the complete graph may be absorbing at some equilibrium. However, if the process of network formation "starts" at the empty network, then the complete graph will never be reached at any equilibrium - only onelink graphs will form. So, this example illustrates the importance of efficient graphs being sustained as strongly absorbing graphs. 
In what follows, we both simplify and extend the logic of inefficient outcomes. The simplification is that we select the equilibrium in question (Theorem 2 applied to all equilibria). But we extend the argument in the sense that we provide a set of conditions (not just an example) under which the complete network is strongly absorbing (for some equilibrium). At the same time, the complete network may be inefficient.

Specifically, we now show that if the network structure satisfies link monotonicity, then the complete graph $\tilde{g}$ can be supported as a strongly absorbing graph at some equilibrium strategy profile. [However, we also give an example to show that the complete graph is not necessarily strongly absorbing at all equilibria.]

Theorem 3 Suppose $(a, w)$ satisfies link monotonicity. Then, for all $\delta \in$ $(0,1)$, there is some equilibrium $\mu^{*}$ such that $\tilde{g}$ is strongly absorbing.

Proof. Consider the strategy profile $\mu^{*}$ where at any principal state $(g, i j)$, $i$ and $j$ form the link $i j$ (if unlinked), and do not sever any existing link in $g$. We show that such $\mu^{*}$ is an equilibrium strategy profile.

It will be sufficient to show that

$$
\text { For all } g \text {, for all } i j \notin g, V_{i}\left(g+i j, \mu^{*}\right)>V\left(g, \mu^{*}\right) \text {. }
$$

We prove that (8) is true by induction on the number of links ("distance") that separates $g$ from $\tilde{g}$. 
If $g$ and $\tilde{g}$ are separated by a single link, then $g=\tilde{g}-i j$. In this case, given the strategies of all players, $i$ and $j$ obtain (in each period) precisely $a_{i}(g, w)$ and $a_{j}(g, w)$ as long as they do not form a link, and $a_{i}(\tilde{g}, w)$ and $a_{j}(\tilde{g}, w)$ if they do. So given link monotonicity, (8) is trivially true in this case.

Next, define $M \equiv\left(\begin{array}{l}n \\ 2\end{array}\right)$. This is the number of all possible pairs, and therefore also the maximal distance between $\tilde{g}$ and any $g$. Suppose, inductively, that for $2 \leq K \leq M$, (8) holds for all $g$ which are at a distance of $K-1$ or less from $\tilde{g}$. Pick any $g$ (with $i j \notin g$ ) at distance $K$ from $\tilde{g}$. Define $g^{\prime} \equiv g+i j$. Then

$$
\begin{aligned}
V_{i}\left(g, \mu^{*}\right) & =\frac{M-K}{M}\left(a_{i}(g, w)+\delta V_{i}\left(g, \mu^{*}\right)\right)+\frac{1}{M}\left(\sum_{k l \notin g}\left(a_{i}(g+k l, w)+\delta V_{i}\left(g+k l, \mu^{*}\right)\right)\right) \\
& =\frac{(M-K) a_{i}(g, w)+a_{i}\left(g^{\prime}, w\right)+\delta V_{i}\left(g^{\prime}, \mu^{*}\right)+\sum_{k l \notin g^{\prime}}\left(a_{i}(g+k l, w)+\delta V_{i}\left(g+k l, \mu^{*}\right)\right)}{M-\delta(M-K)}
\end{aligned}
$$

Similarly,

$$
\begin{aligned}
V_{i}\left(g^{\prime}, \mu^{*}\right) & =\frac{(M-K+1) a_{i}\left(g^{\prime}, w\right)+\sum_{k l \notin g^{\prime}}\left(a_{i}\left(g^{\prime}+k l, w\right)+\delta V_{i}\left(g^{\prime}+k l, \mu^{*}\right)\right)}{M-\delta(M-K+1)} \\
> & \frac{(M-K+1) a_{i}\left(g^{\prime}, w\right)+\sum_{k l \notin g^{\prime}}\left(a_{i}(g+k l, w)+\delta V_{i}\left(g+k l, \mu^{*}\right)\right)}{M-\delta(M-K+1)}
\end{aligned}
$$

where the inequality invokes both link monotonicity and the induction hypothesis (noting that for all $k l \notin g^{\prime}, g^{\prime}+k l=\{g+k l\}+i j$, and that $g+k l$ is at a distance of $K-1$ from $\tilde{g}$ ).

Combining (9) and (10), we may conclude that

$[M-\delta(M-K+1)] V_{i}\left(g^{\prime}, \mu^{*}\right)-[M-\delta(M-K)] V_{i}\left(g, \mu^{*}\right)>(M-k)\left[a_{i}\left(g^{\prime}, w\right)-a_{i}(g, w)\right]-\delta V_{i}\left(g^{\prime}, \mu^{*}\right)$, 
so that

$$
V_{i}\left(g^{\prime}, \mu^{*}\right)-V_{i}\left(g, \mu^{*}\right)>\frac{M-K}{M-\delta(M-K)}\left[a_{i}\left(g^{\prime}, w\right)-a_{i}(g, w)\right]>0,
$$

the last inequality following from link monotonicity once again. This completes the inductive step.

Link Monotonicity does not imply that the complete graph is strongly absorbing at all equilibria. In Example 1, it is easy to check that there can be an equilibrium in which the one-link graph is an absorbing graph.

Finally, we turn to a positive result regarding efficiency. In the next theorem, we show that if the network structure satisfies IRL, and if the allocation rule is the component-wise egalitarian rule, then the complete graph will be strongly absorbing at some pure strategy equilibrium profile. A first reaction may be that this is an obvious result. After all, if $\tilde{g}$ is the unique strongly efficient graph, then surely everyone has a common interest in reaching $\tilde{g}$ and then staying there? However, suppose that aggregate payoffs are negative for "small" graphs. Then, all individuals prefer to join the network after it has reached the critical threshold beyond which payoffs are nonnegative. So, it is a non-trivial issue to show that free-riding behaviour does not become so pervasive so as to prevent the network formation process from converging to the complete network.

The following example illustrates the nature of the free-riding behavior when the network structure satisfies IRL. 
ExAmple 3 Let $N=\{1,2,3\}, w(g)=-4$ if $\# g=1, w(\{i j, j k\})=-3, w(\tilde{g})=$ 3. Suppose the allocation rule is the component-wise egalitarian rule.

Then, it cannot be an equilibrium for all pairs to form a link at all networks. For suppose, both 1 and 2 want to form additional links at each opportunity. This then allows 3 to free ride. To check this, let $V_{3}$ denote discounted payoffs if 3 also agrees to form a link at each opportunity. Routine calculations yield (for $i=1,2$ )

$$
V_{3}(\emptyset, 3 i)=-\frac{-6}{3-\delta}-\frac{6 \delta}{(3-\delta)(3-2 \delta)}+\frac{2 \delta^{2}}{(3-\delta)(3-2 \delta)(1-\delta)}
$$

On the other hand, if 3 refuses to form the first link, but is willing to form subsequent links, then the discounted payoff at a principal state $(\emptyset, 3 i)$ is

$$
V_{3}^{\prime}(\emptyset, 3 i)=\frac{\delta^{2}}{(3-2 \delta)^{2}(3-\delta)}\left[-6+\frac{2 \delta}{1-\delta}\right]>V_{3}(\emptyset, 3 i)
$$

Of course, whether free-riding will take place or not depends on the specific parameter values. For instance, if $\delta$ is "close" to 1 , and if $w(\tilde{g})$ is sufficiently large relative to the absolute values of other graphs, then all agents will want to form additional links at each opportunity.

THEOREM 4 Suppose the network structure satisfies IRL. Then, for all $\delta$ sufficiently large, there is an equilibrium strategy profile such that $\tilde{g}$ is strongly absorbing. 
Proof: If a component $c$ is nonsingleton and $w(c) \geq 0$, call it nonnegative. Define a strategy profile $\mu^{*}$ as follows. Consider any state $s=(g, i j)$.

(I) If either $i$ or $j$ (or both) are members of some nonnegative component of $g$ (perhaps different ones), then $i$ and $j$ retain all existing links and form the link $i j$ if it did not exist before (and if $s$ is a principal state).

(II) Otherwise, $i$ and $j$ follow any equilibrium strategy profile in the "restricted" game where strategies follow (I) whenever (I) applies. ${ }^{18}$

Notice that in Case I, the immediate payoffs of both $i$ and $j$ must go up (this is easy to verify using the definition of IRL). This means that link monotonicity is satisfied on the subdomain in which (I) applies. Because $w$ is additive, the behavior specified by (I) is an equilibrium, by Theorem 3. So $\mu^{*}$ is an equilibrium in the overall game.

We will now show that the equilibrium entails convergence to $\tilde{g}$. To this end, we first claim

FACT 1 . If $V_{i}\left(s, \mu^{*}\right)>0$ for any state $s$ and any $i$, the process must converge to $\tilde{g}$ from that state.

To prove this, let $H$ be the set of all graphs that contain at least one nonnegative component. By (I), if the process enters $H$, then it must converge

\footnotetext{
${ }^{18}$ The strategy profile $\mu^{*}$ is well defined because our existence proof implies that an equilibrium will exist in the restricted game.
} 
to $\tilde{g}$ a.s. But if the process does not ever enter $H$, then no player can ever earn a strictly positive payoff, by the definition of IRL. This proves Fact 1.

FACT 2. There exists a $\bar{\delta} \in(0,1)$ such that for all $\delta \geq \bar{\delta}$ and for any graph $g$, there is some stage of the form $s=(g, i j)$ where the active players $i$ and $j$ earn a positive payoff.

To show this, first note that once the process enters $H$, there is a stochastic, bounded time (independent of $\delta$ ) within which the complete graph $\tilde{g}$ will be reached. Notice that $a_{i}(\tilde{g}, w)>0$ for all $i$, so for any $i$,

$$
V_{i}\left(s, \mu^{*}\right) \geq \underline{\mathrm{V}}_{i}(\delta),
$$

for some function $\underline{\mathrm{V}}_{i}(\delta)$ which goes to $+\infty$ as $\delta \rightarrow 1$.

Now, take any connected $\bar{g} \notin H$ such that $\bar{g}+i j \in H$ for some $i j$, and let $s=\left(\bar{g}, i j\right.$ ). Both $i$ and $j$ can get at least $\delta \underline{\mathrm{V}}_{k}(\delta)$ (for $k=i, j$ ) by forming the link $i j$. This means that their equilibrium payoff is strictly positive. It follows from Fact 1 that from $s$ the process must converge to $\tilde{g}$ almost surely.

Let $q \equiv|\{k l \mid(\bar{g}+k l) \in H\}|$. Then the following is immediate: The probability that the network process converges to $\tilde{g}$ from $\bar{g}$ is at least $\frac{q}{M}$.

Next, observe that the (stochastic) time to any pair being active is a bounded random variable, independent of the discount factor. Moreover, any active pair can always break all links. Therefore, there is finite $L$, independent 
of the discount factor, such that for all states $s$ and for all individuals $i$, $V_{k}\left(s, \mu^{*}\right) \geq-L$ no matter what the discount factor is.

Now, take any $g^{\prime}$ and $k l$ such that $g+k l=\bar{g}$, and consider $s^{\prime}=(g, k l)$. Since $k l$ can form the link $k l$, for each $i \in\{k, l\}$

$$
V_{i}\left(s^{\prime}, \mu\right) \geq a_{i}(\bar{g}, w)+\frac{q}{M} \delta^{2} \underline{\mathrm{V}}_{i}(\delta)-\frac{M-q}{M} L
$$

Notice that for sufficiently large values of $\delta, V_{i}\left(s^{\prime}, \mu^{*}\right) \rightarrow \infty$ as $\delta \rightarrow 1$. Therefore the active pair $(k, l)$ enjoys a strictly positive payoff at this stage.

Continuing these arguments inductively, it is possible to establish Fact 2 for all initial networks $g$.

Combining Facts 1 and 2, the proof of the proposition is complete.

The next example shows that "static" coordination failures can occur even when the network structure satisfies IRL - the example shows that $\tilde{g}$ is not strongly absorbing at some equilibrium profiles even though IRL is satisfied.

Example 4 Suppose $N=\{1,2,3,4\}$, the network structure satisfies IRL, with $w(\tilde{g})=4, w(\{i j\})=-100, w(g)<0$ for all other nonempty $g \subset \tilde{g}$. Let the allocation rule be $a^{e}$, and $\delta>\frac{6}{151}$.

Let $\boldsymbol{\mu}$ be such that each pair $i, j$ breaks all links at each $(g, i j)$ and also refuses to form the link ij if $i j \notin g$. This makes the empty graph the strongly 
absorbing graph. To check that this is an equilibrium, we simply check incentives to follow this strategy profile at $\tilde{g}$. First, note that for all $g$ and $i, j$,

$$
V_{i}(g, i j, \boldsymbol{\mu})=0
$$

Suppose $i, j$ deviate at $(\tilde{g}, i j)$ and dont break any links. Denoting this strategy profile by $\boldsymbol{\mu}^{\prime}$, routine calculation yields

$$
V_{i}\left(\tilde{g}, i j, \boldsymbol{\mu}^{\prime}\right)=\frac{6(6-151 \delta)}{(6-\delta)^{2}}<0
$$

This shows that $\boldsymbol{\mu}$ is an equilibrium. 


\section{REFERENCES}

Adserà, A. and D. Ray (1998). "History and Coordination Failure." Journal of Economic Growth 3, 267-276.

Aumann, R. and R. Myerson (1988) "Endogenous Formation of Links Between Players and Coalitions: An Application of the Shapley Value," in A. Roth (ed), The Shapley Value, Cambridge University Press, 175191.

Bala, V. and S. Goyal (2001) "Self-Organization in Communication Networks," Econometrica 68, 1181-1230.

Bandiera, O. and I. Rasul (2002), "Social Networks and the Adoption of New Technology in Northern Mozambique," mimeo., London School of Economics; CEPR Discussion Paper 3341.

Bramoullé, Y. and R. Kranton (2002), "Social Learning, Social Networks, and Search," mimeo., Department of Economics, University of Maryland.

Calvo-Armengo, A. and M. Jackson (2001), "Social Networks and the Resulting Dynamics and Patterns of Employment and Wages," mimeo., CalTech.

Chamley, C., and D. Gale (1994). "Information Revelation and Strategic Delay in a Model of Investment," Econometrica 62, 1065-1085. 
Conley, T. and C. Udry (2002), "Learning About a New Technology: Pineapple in Ghana," mimeo., Department of Economics, Yale University.

Currarini, S. and M. Morelli (2000), "Network Formation with Sequential Demands," Review of Economic Design 5, 229-250.

Dutta, B., and M.O. Jackson (2000), "The Stability and Efficiency of Directed Communication Networks," Review of Economic Design 5, 251272.

Dutta, B., and M.O. Jackson (2003) (edited), Models of the Formation of Networks and Groups, Springer-Verlag.

Dutta, B., and S. Mutuswami (1997) "Stable Networks," Journal of Economic Theory 76, 322-344.

Dutta, B., A. van den Nouweland, and S. Tijs (1998) "Link Formation in Cooperative Situations," International Journal of Game Theory 27, 245-256.

Fafchamps, M. and S. Lund (2000) "Risk-Sharing Networks in Rural Philippines," mimeo., Stanford University.

Genicot, G. and D. Ray (2003), "Group Formation in Risk-Sharing Arrangements," Review of Economic Studies 70, 87-113. 
Goyal, S. and J. Moraga-Gonzalez (2001), "R \& D Networks", Rand Journal of Economics, 32, 686-707.

Goyal, S. and S. Joshi (2003), "Networks of Collaboration in Oligopolies", Games and Economic Behavior, 43, 57-85.

Jackson, M.O. (2001), "The Stability and Efficiency of Economic and Social Networks," in M.Sertel (edited) Advances in Economic Design, (in press).

Jackson, M.O. and A. Watts (2002), "The Evolution of Social and Economic Networks," to appear in Journal of Economic Theory.

Jackson, M.O. and A. Wolinsky (1996), "A Strategic Model of Social and Economic Networks," Journal of Economic Theory, 71, 44-74.

Kariv, S. (2002), "Social Learning in a Network," mimeo., Department of Economics, New York University.

Konishi, H. and D. Ray (2003), "Coalition Formation as a Dynamic Process," Journal of Economic Theory 110, 1-41.

Kranton, R. and D. Minehart (2000), "Competition for Goods in BuyerSeller Networks," Review of Economic Design 5, 301-332.

Kranton, R. and D. Minehart (2001), "A Theory of Buyer-Seller Networks," American Economic Review. 
Myerson, R (1977), "Graphs and Cooperation in Games," Math. Operations Research 2, 225-229.

Page, F.H., Wooders, M.H. and S. Kamat (2001), "Networks and Farsighted Stability", mimeo., Working Paper 621, Department of Economics, University of Warwick.

Slikker, M. and A. van den Nouweland (2000), "Network Formation Models with Costs for Establishing Links," Review of Economic Design 5, 333-362.

Slikker, M. and A. van den Nouweland (2001), "A One-stage Model of Link Formation and Payoff Division", Games and Economic Behavior 34, 153-175.

Tesfatsion, L. (1997) "A Trade Network Game with Endogenous Partner Selection," in H. Amman et al (eds.), Computational Approaches to Economic Problems, Kluwer Academic Publishers, 249-269.

Tesfatsion, L. (1998) "Gale-Shapley matching in an Evolutionary Trade Network Game," Iowa State University Economic Report no. 43.

Wang, P. and A. Watts (2002), "Formation of Buyer-Seller Trade Networks in a Quality-Differentiated Product Market," mimeo., Southern Illinois University.

Wasserman, S. and K. Faust (1994) Social Network Analysis: Methods and Applications, Cambridge University Press. 
Watts, A. (2001) "A Dynamic Model of Network Formation," Games and Economic Behavior 34, 331-341.

* Complete list of working papers is available at the CDE website: http://www.cdedse.org/worklist.pdf 